\title{
Empfehlungen für Evaluationsstudien von Informationssystemen im Gesundheitswesen
}

\author{
E. Ammenwerth ${ }^{a}$, M. D. Denz ${ }^{b}$
}

Die Verbreitung von Informationssystemen im Gesundheitswesen ist im Zunehmen begriffen. Hoffnungsvolle Erwartungen beruhen auf der Annahme, dass durch den Einsatz von Informationssystemen eine qualitative Verbesserung des Gesundheitswesens erzielt werden sollte, ebenso werden ökonomische Einsparungen erwartet. Ob diese Erwartungen tatsächlich erfüllt werden, ist noch offen. Die Unterscheidung zwischen Anspruch und Wirklichkeit hängt davon $a b$, dass Informationssysteme und deren Auswirkungen nach wissenschaftlichen Kriterien evaluiert werden. Die Empfehlungen der internationalen Expertengruppe HIS-EVAL sollen dazu beitragen.

a Ass.-Prof. Dr., Forschungsgruppe Bewertung von Informationssystemen des Gesundheitswesens, Universität für Medizinische Informatik und Technik Tirol (UMIT), Innsbruck, Österreich

b Dr. med., Leiter Abteilung eHealthcare, FMH, Bern, Lehrbeauftragter UMIT

Korrespondenz:

Ass.-Prof. Dr. Elske Ammenwerth Universität für Medizinische Informatik und Technik Tirol (UMIT) Forschungsgruppe Bewertung von Informationssystemen des Gesundheitswesens

Innrain 98

A-6020 Innsbruck

E-Mail: elske.ammenwerth@umit.at

\section{Hintergrund}

Das Gesundheitswesen ist ohne die systematische Verarbeitung von Informationen nicht mehr denkbar. Informationstechnologie hat in den letzten Jahren eine weite Verbreitung in Krankenhäusern und im ambulanten Bereich gefunden. Informationstechnologie hat offenbar das Potential, die Effizienz von Abläufen zu erhöhen und dadurch indirekt die Patientenversorgung zu verbessern. Allerdings birgt der Einsatz von Informationstechnologie auch Gefahren und Probleme. So können unzureichend gestaltete Werkzeuge die Benutzer in ihrer Arbeit behindern, und Softwarefehler können zu Datenverlust oder sogar zur Schädigung von Patienten führen.

Daher ist es sehr wichtig, die Qualität der Informationstechnologie und ihre Auswirkungen systematisch zu überwachen und zu verbessern. Die Evaluation der Informationstechnologie kann helfen, Probleme rechtzeitig zu erkennen und schnell zu lösen. Dies führt letztendlich zu einer höheren Kosteneffizienz von Informationstechnologie und zu einer verbesserten Versorgung.

Die Evaluation von Informationstechnologie erfordert die geeignete Kombination von Methoden z. B. aus den Sozialwissenschaften, der Informatik und der Biometrie. Der interdisziplinäre Austausch ist aber häufig nicht optimal ausgeprägt. Zur Förderung einer interdisziplinären Diskussion über die Weiterentwicklung der Evaluationsforschung wurde daher im April 2003 an der Privaten Universität für Medizinische Informatik und Technik ein Europäischer
Symposium «Evaluation von Informationssystemen» am Kongress eHealthCare.ch Im Rahmen des Kongresses eHealthCare.ch, der am 16. und 17. Oktober 2003 an der Universität Irchel in Zürich stattfinden wird, ist das Symposium 18 dem Thema «Evaluation von Informationssystemen» gewidmet. Frau Prof. Ammenwerth wird eine Einführung in aktuelle Konzepte und Methoden der IT-Evaluation geben, ebenso über die Resultate der Expertengruppe HIS-EVAL berichten. In der anschliessenden Diskussion wird die Möglichkeit angeboten, praxisbezogene Fragen und Erfahrungen auf dem Hintergrund der HIS-EVAL-Empfehlungen zu vertiefen. Dabei sollen nicht nur Klinikinformationssysteme berücksichtigt werden, sondern auch Praxisinformationssysteme und andere im Gesundheitswesen eingesetzte Informationssysteme.

Workshop «New Approaches to the Systematic Evaluation of Health Information Systems (HISEVAL)» organisiert. Der Workshop wurde von der European Research Foundation (ESF) finanziert. Die Ziele des Workshops waren:

- Zusammenbringen von Experten aus verschiedenen Bereichen wie Informatik, Medizinische Informatik, Ökonomie, Gesundheitswesen, Biometrie, Psychologie und Soziologie.

- Ermöglichung der Diskussion und des Austauschs zu den dringenden Themen der Evaluationsforschung zwischen verschiedenen Fachbereichen sowie Ermöglichung des Austauschs von Wissen über Methoden und Werkzeugen.

- Planung konkreter internationaler und interdisziplinärer Initiativen wie die Entwicklung von Leitlinien oder Methodendatenbanken zur Unterstützung von Evaluationsstudien.

- Aufbau eines Europäischen Netzwerkes zur Unterstützung langfristiger Forschungsaktivitäten. 


\section{Ablauf des Innsbrucker Workshops}

Insgesamt nahmen an dem Workshop 23 speziell ausgewählte Forscher aus 10 Europäischen Ländern teil. Der Workshop umfasste eine Reihe von Arbeitsgruppensitzungen zu ausgewählten Themen sowie verschiedene Plenarsitzungen. Das Programm war in drei wesentliche Teile gegliedert:

1. Was sind die Probleme und Barrieren bei der Evaluation von IT im Gesundheitswesen?

2. Was können Visionen und Strategien zur Verbesserung der Evaluationsforschung sein?

\section{Empfehlungen}

1. Evaluation erfordert eine ausreichende Finanzierung. Umfassende Evaluationsstudien sind nur möglich, wenn ausreichend Geldmittel hierfür bereits bei der Planung eines Projekts alloziert werden.

2. Die Terminologie für Evaluationsstudien soll vereinheitlicht werden.

3. Evaluationsstudien sollen methodisch sauber durchgeführt werden. Nur so sind ihre Ergebnisse nachvollziehbar und glaubwürdig.

4. Die Auswahl von Evaluationsmethoden soll sich ausschliesslich an der jeweiligen Fragestellung orientieren, und nicht z. B. an dem professionellen Hintergrund des jeweiligen Projektleiters.

5. Die Weiterentwicklung von Evaluationsmethoden soll stärker gefördert werden, entsprechende Publikationen sollten von Fachzeitschriften ermuntert werden.

6. Evaluatoren sollten frei von externem Druck sein. Nur wenn die Studie sich ausschliesslich auf die Fragestellung konzentrieren kann, werden ihre Ergebnisse valide und hilfreich sein.

7. Leitlinien zur Planung und Durchführung von Evaluationsstudien sind zu entwickeln und zu verbreiten.

8. Evaluationsmethoden sollten regelmässiger Bestandteil der Ausbildung in Medizinischer Informatik werden.

9. Die Einrichtung von Evaluationsnetzwerken zum interdisziplinären Austausch von Wissen und Ergebnissen ist zu fördern.

10. Eine Informationsstelle zur Dokumentation geplanter, durchgeführter oder abgeschlossener Evaluationsstudien ist einzurichten.
3. Was können konkrete zukünftige Aktivitäten zur Verbesserung der Evaluationsforschung sein?

\section{Die Deklaration von Innsbruck}

Neben einem intensiven fachlichen Austausch und der Planung konkreter zukünftiger Aktivitäten wurde von den Teilnehmern die Deklaration von Innsbruck erarbeitet, welche in Kürze breit publiziert werden soll. Diese Deklaration benennt zunächst die wesentlichen Beobachtungen und Einschätzungen zur Evaluationsforschung. Die Deklaration umfasst dann im Hauptteil eine Reihe von Empfehlungen an Forscher, Praktiker, Entscheidungsträger und Politiker. Sie will so helfen, das Bewusstsein für den Nutzen, aber auch die Komplexität von Evaluationsstudien zu steigern und sie will konkrete Wege und Aktivitäten aufzeigen, um die Evaluationsforschung in Zukunft effizienter zu gestalten. Die wesentlichen Empfehlungen seien im Folgenden verkürzt dargestellt:

Die Teilnehmer des Workshops initiierten ausserdem den Aufbau eines Netzwerks für Evaluationsforschung, der derzeit mehr als 100 Mitglieder aus ganz Europa umfasst. Der Informationsaustausch in diesem Netzwerk erfolgt derzeit überwiegend per E-Mail. Ein Mailverteiler wurde eingerichtet, in den sich alle Interessierten eintragen können. Mehr Informationen hierfür sind unter http://www.umit.at/mailman/ listinfo/eval abrufbar. Weiterhin wird derzeit an der Erarbeitung von Leitlinien für die Durchführung von Evaluationsstudien sowie an Leitlinien für die Publikationen von Studien gearbeitet. Erste Ergebnisse sind bis Ende 2003 zu erwarten. Die definitiven Empfehlungen sollen in den führenden wissenschaftlichen und Fachzeitschriften veröffentlicht werden.

\section{Fazit und Ausblick}

Der Innsbrucker Workshop hat die Notwendigkeit einer verstärkten internationalen und interdisziplinären Zusammenarbeit im Bereich der Evaluation von Informationstechnologie im Gesundheitswesen deutlich gemacht. Wir hoffen, dass dieser Workshop den Kern für ein Europäisches Netzwerk gelegt hat, welcher die Evaluationsforschung voranbringen kann, die Qualität von Informationstechnologie verbessern hilft und dadurch letztendlich auch zu einer Verbesserung der Patientenversorgung beitragen wird. 


\section{Danksagung}

Wir möchten uns bei allen Teilnehmern des Innsbrucker Workshops für ihre wertvollen Beiträge bedanken. Die Teilnehmenden waren: Jos Aarts, Elske Ammenwerth, Andrea Berghold, Marie-Catherine Beuscart-Zéphir, Jytte Brender, Thomas Bürkle, Martina Deibl, Martin Denz, Rolf Holle, Mathew Jones, Joe Liu, Christian Nohr, Ulrich Prokosch, Michael Rigby, Cornelia Ruland, Heike Sander, Nikki Shaw, Arjen Stoop, Jan Talmon, Vivian Vimarlund, Jeremy Wyatt sowie Frieda Kaiser und Gudrun Hübner-Bloder. Dank auch an die ESF für die Finanzierung des Workshops und an Hui Wang und Karl-Peter Pfeifer für ihre Unterstützung.

\section{Literatur}

- Friedman C, Wyatt JC. Evaluation Methods in Medical Informatics. New York: Springer; 1997.

- Anderson JG, Aydin CE, Jay SJ (eds.). Evaluating Health Care Information Systems - Methods and Applications. London, New Delhi: Sage Publications; 1994.

- van Gennip E, Talmon J (eds.). Assessment and evaluation of information technologies in medicine. Studies in Health Technology and Informatics. Vol. 17. Amsterdam: IOS Press; 1995.

- Wyatt J, Wyatt S. When and how to evaluate health information systems? Int J Med Inform 2002;69:251-9.

\section{eHealthCare.ch 03}

Neue Technologien und IT in Medizin und Gesundheitswesen

\section{Konferenz und Fachmesse}

Universität Zürich-Irchel, 16./17. Oktober 2003

Schwerpunkt: Der Patient im Informationszeitalter

www.eHealthCare.ch

Leading Mediapartner: EMH Schweizerischer Ärzteverlag AG 